\title{
Die keusche Penelope und der zornige Odysseus: Kursieren einer tragikomischen Opernhandlung zwischen Wien und Norditalien
}

\section{The Chaste Penelope and the Angry Odysseus: Course of a Tragicomic Opera Action between Vienna and Northern Italy}

\author{
Livio Marcaletti / liviomarcaletti@alice.it \\ Institute of Musicology, University of Vienna, AT
}

\begin{abstract}
In the 1710s and 1720s, librettist Pietro Pariati and composer Francesco Bartolomeo Conti cultivated the highly specific genre of tragicommedia per musica at the Habsburg court in Vienna. Of particular interest is their tragicomedy Penelope (1724), which depicts Ulysses as a ridiculous hero guided by his furious jealousy through a comedy of errors where mistaken identity and buffo servants play a relevant role. Far from being new, this tragicomic interpretation of UIysses's return to Ithaca belonged to a long-running tradition: Nicolò Minato's Penelope (Vienna 1670) inspired Matteo Noris's Penelope la casta (Venice 1685), on which Pariati in turn based his spoken tragicomedy La casta Penelope (Milan 1707) and the 1724 Viennese tragicommedia per musica. The present paper focuses on the dramaturgical evolution of this plot and shows a case study of bidirectional librettistic influence along the Vienna-Venice axis.
\end{abstract}

\section{Keywords}

Tragicomedy, Penelope, Ulysses, Nicolò Minato, Matteo Noris, Pietro Pariati, Venetian opera, Viennese opera 
Die Verbreitung der italienischen Oper aus der Apenninenhalbinsel nordwärts ist ein Phänomen, dessen Relevanz für die Etablierung eigener lokaler Produktionen kaum genug betont werden kann. ${ }^{1} \mathrm{Zu}$ diesem Prozess trug nicht nur der Austausch von Libretti und (seltener) deren Partituren bei, sondern auch die Auswanderung von Librettisten, Komponisten, SängerInnen, Instrumentalisten, Bühnenbildnern und anderen Professionellen, die ein italienischsprachiges Kulturprodukt zum europäischen Phänomen verwandelt haben. Ein Muster stellt der Fall des Wiener Kaiserhofes im 17. und 18. Jahrhundert dar. Er verband die Produktion und Rezeption von italienischer Kultur in literarischer wie auch musikalischer Form: Nicht nur wichtige Musiker, sondern auch die bekanntesten italienischen Librettisten des späten 17. und 18. Jahrhunderts wurden nach Wien berufen - Nicolò Minato, Apostolo Zeno, Pietro Pariati und Pietro Metastasio. ${ }^{2}$

Während die meisten derzeitigen Studien im literarwissenschaftlichen und musikwissenschaftlichen Bereich den Kulturtransferphänomenen von Italien nach Norden große Aufmerksamkeit schenken, ${ }^{3}$ wird die entgegengesetzte Richtung ausgehend von den deutschsprachigen Ländern gen Süden weniger berücksichtigt. Im Fokus des vorliegenden Beitrags steht darum das Kursieren einer auf den letzten Gesängen der Odyssee basierenden Opernhandlung: Er dient als Fallstudie der Zirkulation von Libretti und damit verbundener opernästhetischer Tendenzen zwischen Wiener Kaiserhof und norditalienischen Theatern. Gegenstand dieser Untersuchung sind die folgenden vier Opernund Theaterwerke: Penelope von Nicolò Minato (Wien 1670), Penelope la casta von Matteo Noris (Venedig 1685), Pietro Pariatis Theaterstück La casta Penelope (Mailand 1707) und dessen tragikomische Oper Penelope (Wien 1724). Die enge Beziehung zwischen diesen vier Stücken für die Musiktheaterbühne stellt nicht nur einen aufschlussreichen Fall der Verbreitung von Opern und Theaterwerken dar. Vielmehr verdeutlicht sie auch gattungsbezogener Spezifika der tragicommedia per musica, welche die Wiener Opernproduktion der 1710er und 1720er Jahre charakterisierte. In ihrer ständigen Durchdringung und Verbindung von ernster und komischer Ebene weist sie Kontinuitätselemente mit der wienerischen sowie der venezianischen Oper des 17. Jahrhunderts auf.

\section{Die erste Penelope (Wien 1670)}

Nicolò Minato (ca. 1625-1698) war "wohl - was den Umfang und die Vielfalt seines Werkes betrifft - der bedeutendste italienische Hofdichter in Wien ". ${ }^{4}$ Nach fast zwanzig Jahren in Ve-

1 Siehe BIANCONI, Lorenzo. Music in the Seventeenth Century, Cambridge: Cambridge University Press, 1986, S. 220: „The diffusion of Italian opera north of the Alps and the establishment of more or less autonomous local and/or national operatic traditions is a phenomenon of eminent importance for the musical and cultural life of Europe during the second half of the seventeenth century".

2 Siehe NOE, Alfred. Die italienische Literatur in Österreich, Wien: Böhlau Verlag, 2011.

3 Siehe etwa MURARO, Maria Teresa (Hrsg.), L'opera italiana a Vienna prima di Metastasio, Firenze: Olschki, 1990; COLAS, Damien und DI PROFIO, Alessandro (Hrsg.), D'une scène à l'autre, l'opéra italian en Europe, 2 Bde., Wavre, Mardaga, 2009.

4 NOE, Alfred. Nicolò Minato Werkverzeichnis, Wien: Der österreichischen Akademie der Wissenschaften, 2004, S. 7. 
nedig als Librettist und Impresario wurde er im Jahr 1669 als Nachfolger von Aurelio Amalteo nach Wien berufen. Penelope zählt damit zur Gruppe der frühen Libretti, die Minato für den Kaiserhof schrieb und das Antonio Draghi vertonte. Dieses dreiaktige Dramma per musica wurde am 18. November 1670 zum Geburtstag der Kaiserin Eleonora erstmals aufgeführt. Der Auftritt der Erzherzogin Maria Anna Josepha als Tänzerin im abschließenden Ballett bedingte die Verwendung komischer Intermezzi anstatt der sonst üblichen Ballette zwischen den Akten. So wird bereits im Vorwort ankündigt (" $p o$ iché dove s'hanno a intrecciar l'orme del piede d'una serenissima arciduchessa, non doveva precedere scherzo d'altre piante"): ${ }^{5}$ In diesem bestimmten Fall ist darum die Verstärkung der komischen Komponente der Oper durch Intermezzi Ergebnis einer anlassbedingten Entscheidung. ${ }^{6}$

Vorlage der Penelope ist die Erzählung der Rückkehr von Odysseus nach Ithaka zufolge den letzten Gesängen der Odyssee. Auf dieser Basis konstruiert Minato eine eigene Fassung des Mythos, nicht zuletzt durch das Hinzufügen erfundener Figuren und entsprechender Nebenintrigen:

Akt I.: Ulisse kehrt mit seinem Diener Lippio nach Ithaka zurück. Er hat den Palast kaum erreicht, dass er den Freier Acrisio unter dem Fenster Penelopes ein Lied singen sehen. Mittlerweile kommt auch Lippio vorbei und erkennt in der dunklen Nacht seinen Herrn nicht; aus Versehen übergibt Lippio Acrisio das Bild Penelopes, woraufhin dieser denkt, dass seine Liebe durch Penelope erwidert wird. Voll Eifersucht beschließt Ulisse, seine Identität zu verbergen und sich als Alceste auszugeben. Dazwischen wird Penelopes Weblist von ihrem Vater Icario enthüllt, sie will jedoch die Aufforderung ihres Vaters, einen neuen Gatten zu heiraten, nicht berücksichtigen. Die attische Prinzessin Orisbe, die in Männerkleidung ihrem Geliebten Acrisio gefolgt ist, bittet Penelope ihr zu helfen, ihren Gatten zurückzugewinnen. Penelope trifft Ulisse und Lippio und erkennt ihren Gatten, der sich allerdings als Trojaner ausgibt und mit Eifersucht Zuschauer der Werbungen der Freier um Penelope ist, die alle abweist.

Akt II.: Orisbe setzt sich mit Acrisio auseinander, der wegen des Bildes überzeugt ist, Penelope liebe ihn. Penelope verteidigt sich gegenüber Orisbes Anklage, sie hintergangen zu haben: Nachdem Penelope das Bild von Acrisio wiedergewonnen hat, überreicht sie es Orisbe, die dadurch Acrisio eifersüchtig macht, Penelope liebe jetzt Olmiro. Ulisse, der die Szene zwischen Penelope und Orisbe eifersüchtig beobachtet hat, verkündet seiner Frau den Tod ihres Gatten. Die trauernde Königin wird von Lippio getröstet, indem er ihr die wahre Identität des Alceste verrät. Die Königin beschließt nun, Odysseus für seine Eifersucht zu bestrafen.

Akt III.: Als Orisbe/Olmiro als neuer Ehemann der Penelope vorgestellt wird, entschließt Odysseus sich dazu, mit dem Sohn Telemaco und zusammen mit den anderen Freiern Rache an seiner Frau zu nehmen. Dies wird jedoch durch die Aufdeckung der wahren Identität Oris-

5 MINATO, Nicolò. Penelope, Wien: Cosmerovio, 1670, Vorwort: „[...] weil wo sich die Fußstapfen einer durchlauchtigsten Erzherzogin verflechten, darf keine Spielerei anderer Füße zuvorkommen“.

6 Siehe SEIFERT, Herbert. Die Oper am Wiener Kaiserhof im 17. Jahrhundert, Tutzing: Schneider, 1985, S. 226-7. 
bes verhindert, woraufhin Penelope und Odysseus sich versöhnen. Das glückliche Ende ist mit der Aussöhnung von Orisbe und Acrisio besiegelt.

Minato verleiht seinem Libretto eine tragikomische Stimmung vor allem durch zwei Elemente, und zwar den lustigen Diener Lippio sowie das eifersüchtige, eines epischen Helden unwürdige Verhalten von Ulisses.

Lippios Komik kennzeichnen typische stilistische Merkmale der Wiener Libretti Minatos. Sie basiert maßgeblich auf Wortwitzen, die sich oft aus Lippios Missverständnis der gehobenen, dichterischen Sprache ernster Figuren ergeben. Wenn etwa Ulisse das Boot "nuotante abete" (schwimmende Tanne, eine typische Synekdoche) und das Porträt "effigie" (ein dichterisches Wort, das dem Alltagjargon enthoben ist) nennt, ist Lippio völlig verwirrt und antwortet derart: "Sempre, in mal hora, / cosi parlar volete? / Ditemi nave, e non nuotante abete " (Immer so, verdammt! Wollen Sie so mit mir sprechen? Sagen Sie mir Boot und nicht schwimmende Tanne!). Solche Wortspiele sind vergleichbar mit denen der komischen Figur des Gracioso ${ }^{7}$ aus der spanischen comédia. Zu erklären ist diese Ähnlichkeit mit den inzwischen klar belegbaren Einflüssen des spanischen Theaters auf die italienische Oper des 17. Jahrhunderts. ${ }^{8}$

Lippio gibt sich zwar als ignorant, er kann sich aber trotzdem im Notfall schlau verhalten. Nachdem er Penelopes Porträt der falschen Person übergeben hat und Ulisse es fordert, nützt Lippio bewusst seine Ignoranz als Ausrede, um sich der Bestrafung zu entziehen: Er gibt vor, das Wort „imago“ (Bild) nicht zu verstehen und mit „mago “ (Zauberer) zu verwechseln. Ulisses Antwort, „Sciocco: rider mi fai anco tra l'ire” (Albern! Auch in meinem Zorn bringst du mich zum Lachen), ist regelrecht metatheatralisch. Ulisse wird der Streich gespielt, doch er reagiert wie das Publikum, das den Witz lustig finden soll. In diesem Zusammenhang ist ergänzend Lippios bewusste Untertreibung des fehlenden Porträts zu erwähnen: Trotz seiner geringen sprachlichen Fähigkeiten vermag er eine pseudo-philosophische Sprache zu verwenden, um sich die Mühe zu sparen, das Porträt abzuholen:

$$
\text { [...] che tanto }
$$

De l'effigie ti preme?

Fai torto al naturale:

Sì vicino gli sei, e sembra apunto,

Che tu d'aver più stimi (e mi perdona,

Se col mio dir ti spiaccio)

In mano la bugia, ch'il vero in braccio.

7 KINTER, Barbara. Die Figur des Gracioso im spanischen Theater des 17. Jahrhunderts, München: Wilhelm Fink Verlag, p. 52: Das Wortspiel der komischen Sinnverzerrung gibt sich als 'Mißverständnis' aus: Wörter, die im Milieu der Ungebildeten nicht gebräuchlich sind, werden lautlich soweit verändert, bis ein verständlicherer und damit komisch-falscher - Begriff herauskommt.

8 Unter den neusten Beiträgen zum Thema siehe La comedia nueva e le scene italiane nel Seicento (Fausta Antonucci und Anna Tedesco hg.), Firenze: Olschki, 2016. 
[...] warum

kümmerst du dich um das Bild?

Das ist unrecht angesichts des Originalen:

Du bist nun so nah, und trotzdem scheint,

dass du (entschuldige, wenn meine Worte dir leidtun)

lieber die Lüge als die Wahrheit im Arm halten willst.

Komischerweise beherrscht Ulisse dieses Mal das Wort „effigie”, das er zuvor nicht einmal verstanden hatte, problemlos. Solche Wortspiele kommen oft in Minatos Wiener Libretti vor und entsprechen vermutlich einer Vorliebe des höfischen Publikums für harmlose Wortspiele, wobei die in Venedig beliebten erotischen Wortwitze am würdevollen Hof der frommen Kaiserfamilie undenkbar gewesen wären.

Während die Präsenz komischer Dienerrollen in Anlehnung an Lope de Vega eher als eine Osmose des Tragischen und des Komischen denken lässt, ${ }^{9}$ gelangt man erst durch die Rolle des Ulisses zu einer tatsächlichen Durchdringung der zwei Ebenen. Anders als in der Odyssee, sowie etwa in der dem Epos treueren Oper Il ritorno di Ulisse in patria von Badoaro und Monteverdi, wirkt Minatos Ulisse zornig, eifersüchtig und gewalttätig. Er erscheint als Parodie eines Helden, der ungeachtet seiner sprichwörtlichen Schläue nicht hinreichend aufmerksam ist, um seiner Gattin auf die Schliche zu kommen und ihrer Treue Glauben zu schenken. Als Penelope durch Lippio die wahre Identität des verkleideten Ulisse erfährt, revanchiert sie sich bei ihm für sein Misstrauen und verspottet ihn. Die folgende Szene parodiert die Szene der Erkennung Ulisses (Akt III, 1):

Ul.: Penelope.

Pen.: $\quad$ Sei qui? Che vuoi?

Ul.:

Amore

Sì di leggier t’impresse

L'imagin mia nel seno,

Che più non mi conosci?

Pen.: Che vaneggi?

Ul.: $\quad$ E me vivo.

Ad amori ti pieghi?

A' sponsali t'accingi?

Io son Ulisse.

Pen.: $\quad$ Che follie che fingi?

Mostra di partire: egli la ferma.

Ul.: Così accogli il consorte?

Pen.: Falce di cruda morte

L'accolse.

Ul.: $\quad$ Non è ver: son io, ti dico.

Pen.: Eh ch'il mio sposo è un re, non un mendico.

9 GIGLIUCCI, Roberto. Tragicomico e melodramma, Milano: Mimesis Edizioni, 2011, S. 36. 
Ul.: Inventai questa frode,

Acciò di penetrar, s'io son tradito,

Meglio le vie m'aprisse.

Pen.: Eh con inganno non verrebbe Ulisse.

Ul.: Se coperte da gl'anni

De le prime sembianze

Così rimangon l'orme,

Che più non le ravisi,

Se non conosci i lumi,

Non figuri gl'accenti, e se non credi

Ai miei detti veraci,

Conoscerai i baci.

Va per baciarla; ella lo scaccia.

Pen.: Scostati; che d'Olmiro

Moglie son io.

Ul.: $\quad$ Troppo ostinata sei

Nel concepito inganno.

Pen.: (Sopporta pur: tuo danno).

Ul.: Penelope.

Pen.: Bist du da? Was willst du?

Ul.: Die Liebe hat mein Bild in deine Brust so leicht aufgeprägt, dass du mich nicht mehr erkennst?

Pen.: Was phantasierst du?

Ul.: Und während ich noch lebe, gibst du neuen Lieben nach? Bereitest du dich für eine neue Hochzeit vor? Ich bin Ulisse.

Pen.: Was für einen Wahnsinn machst du vor?

Sie zeigt, abzugehen: Er hält sie an.

Ul.: Dermaßen empfingst du deinen Gatten?

Pen.: Der grausame Tod mit seiner Sense nahm ihn auf.

Ul.: Das ist nicht wahr: Ich sage dir, ich bin's.

Pen.: Hä, mein Gatte ist kein Bettler, sondern ein König.

Ul.: Ich habe diese List erfunden, um ins Palast sicherer durchzudringen, wäre ich verraten gewesen.

Pen.: Hä, Ulisse käme ohne List.

Ul.: Sind die Spuren meiner älteren Züge des Alters wegen nicht länger erkennbar, erkennst du meine Augen, meine Stimme nicht wieder, und glaubst du nicht an meine wahrhaften Ausdrücke, dann wirst du meine Küsse erkennen.

Er versucht, sie zu küssen; sie jagt ihn fort.

Pen.: Weg von mir; ich bin Gattin des Olmiro.

Ul.: Du beharrst auf die ausgedachte List allzu viel.

Pen.: (Du musst erdulden, du bist selber Schuld). 
In der Odyssee erfolgt die Erkennung des Odysseus dadurch, dass er die Fangfrage der misstrauischen Penelope über ihr auf einem Baumstumpf fixiertes Ehebett richtig beantwortet - ein Umstand, von dem kein Betrüger wissen konnte. Bei Minato scheitert zuerst die Agnition; nicht, weil Penelope nicht an Ulisse glaubt (Lippio hat schon dessen Identität verraten), sondern aus Rache. Die misslungene Wiedererkennung wird zur Verspottung von Ulisses. Besonders ironisch klingt Penelopes Behauptung, „Ulisse käme ohne List", da Odysseus bekanntlich ein Meister der Schlauheit ist (in diesem Zusammenhang sei nur an die List des Trojanischen Pferdes erinnert). Ulisse reagiert dann regelrecht grotesk als schnulziger Liebhaber, der sich durch Küsse erkennen lassen will: ein Verhalten, das keineswegs dem epischen Odysseus entspricht. Auch im letzten Akt wirkt Ulisse wie eine Parodie der originalen Figur in der Odyssee. Nachdem Orisbe/ Olmiro sich als neuen Gatten Penelopes ausgibt, mündet Ulisses Rache in die paradoxale Entscheidung, sich mit den Freiern zusammenzuschließen, um mit ihrer Hilfe seinen eigenen Palast zu stürmen und das neue Paar zu töten. Die Auseinandersetzung zwischen dem Ulisse furioso und seiner kaltblütigen, spöttelnden Gattin erreicht schließlich tragikomische Spitzen, zu denen zusätzliche Kommentare von Lippio mit seinen Verständigungsproblemen ${ }^{10}$ beitragen:

Uli.: Quest'è velen, sleale,

Bevilo.

Lip.: $\quad$ Che non dir succo letale!

Pen.: Oh sei troppo sdegnoso!

Che feci poi di mal, prendermi un sposo!

Uli.: Das ist Gift, Treuloser,

Trink es.

Lip.: Warum nennst du es nicht tödlichen Saft?

Pen.: Ach, du bist ja zu schnippisch!

Was habe ich Böses getan? Ich habe nur meinen Bräutigam gewählt!

Eine solche Transformation der Odyssee wird für gewöhnlich als Parodie bezeichnet. Nach Gérard Genettes präziser Klassifikation der hypertextuellen Transformationen mit einer „satirischen Funktion“ erscheint jedoch die Kategorie der burlesken Travestie angemessener. So definiert Genette jene Transformationen, bei denen das Sujet des Hypotextes beibehalten wird, als stilistisch erniedrigt. ${ }^{11}$ Durch diese Erniedrigung (dévalorisation) der Heldenfiguren von Ulisse und Penelope erfolgt eine Entmythisierung (démistification) der originalen Handlung der Odyssee. Darum kann Minatos Penelope als Tragikomödie bezeichnet werden. In dieser sprechen und agieren Penelope und vor allem Ulisse auf eine Weise, die des Epos nicht würdig erscheint.

10 Das Wortspiel basiert auf einer vorausgehenden Szene, bei der das Wort „succo letale“ (tödlicher Saft) als erhabeneres Synonym für „veleno“ (Gift) verwendet wurde.

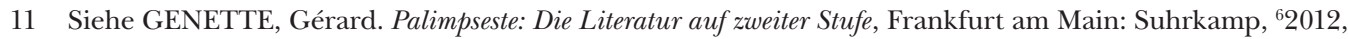
Kap. VII und XII. 


\section{Von Minatos Penelope zu Pariatis Tragikomödien La casta Penelope (Mailand 1707) und Penelope (Wien 1724) durch Noris' Penelope la casta (Venedig 1685)}

Der Vergleich von Minatos Libretto mit der 1685 im Theater S. Giovanni Grisostomo in Venedig aufgeführten Penelope la casta (Libretto von Matteo Noris, Vertonung von Carlo Pallavicino) zeigt etliche Gemeinsamkeiten, obschon Noris keinen Zusammenhang mit der Wiener Penelope eingesteht. Es ist bekannt, dass Minatos Libretti in Italien (und dort auch in Venedig) kursierten und häufig aufgeführt wurden, wenngleich zumeist mit neuen Vertonungen, da handschriftliche Partituren nicht so einfach wie Drucklibretti verbreitet werden konnten. ${ }^{12}$ Da mehrere für den Wiener Kaiserhof geschriebene Libretti in Venedig rezipiert und neu vertont wurden, ist es nicht unwahrscheinlich, dass ein Druckexemplar von Penelope in Noris' Hände gelangt sein könnte.

Die Dramatis personae zeigt Ähnlichkeiten und Unterschiede mit Minatos Libretto. Noris lässt insgesamt achtundzwanzig Figuren spielen, also doppelt so viele wie in der Wiener Penelope. Dies liegt auch darin begründet, dass Noris parallele Szenen am Ende jedes Aktes und am Anfang der ganzen Oper hinzufügt, in denen allegorische Figuren wie etwa das Mögliche, das Unmögliche, der Zweifel, die Wahrheit, der Verdacht usw. die Haupthandlung in autonomen Szenen kommentieren, ohne sich direkt einzumischen. Aus diesem Grund konnten sie in späteren Wiederaufnahmen mit neuer Vertonung (z. B. jene von Alessandro Scarlatti am Teatro S. Bartolomeo in Neapel, 1696) ohne weitere Anpassungsmaßnahmen gestrichen werden. Wenn man die allegorischen Figuren tilgt, zeigt der Kern der Handlung viele Ähnlichkeiten mit der vermutlichen Vorlage, wie das folgende Schema es zusammenfassend verdeutlicht:

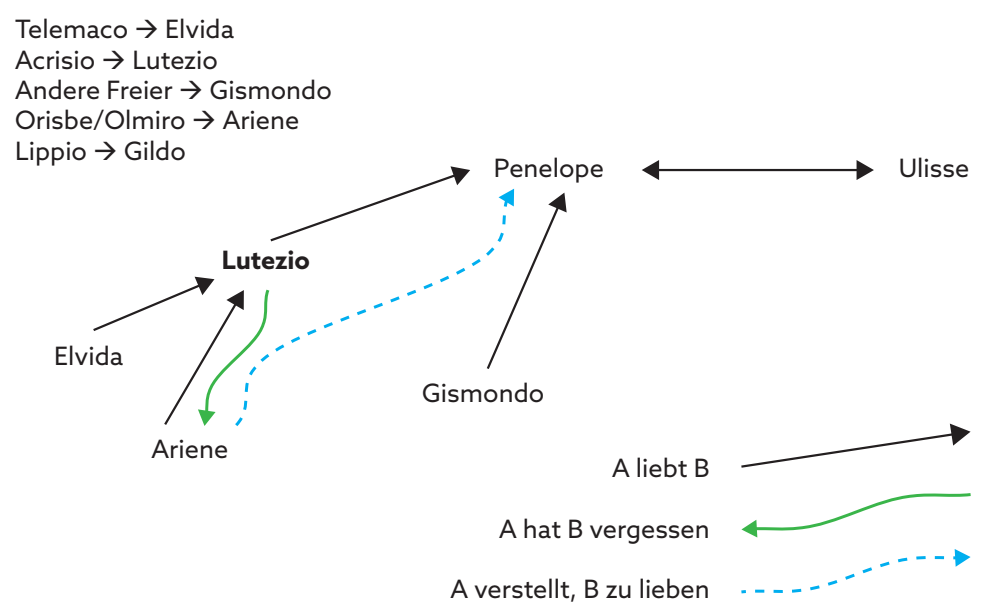

Abb. 1 Ein Schema der Beziehungen zwischen allegorischen Hauptfiguren

12 DUBOWY, Norbert. Opere di Draghi in Italia? In „Quel novo Cario, quel divin Orfeo”: Antonio Draghi da Rimini a Vienna; atti del convegno internazionale, (Rimini, Palazzo Buonadrata, 5-7 ottobre 1998). Davide Daolmi - Emilio Sala (Hrsg.). Lucca: LIM, 2000, S. 226. 
Gemeinsame Figuren der beiden Libretti sind nicht nur Ulisse mit seinem eifersüchtigen und anti-heroischen Verhalten, sondern auch der Diener und das zweite Liebespaar: Freier und verlassene Verlobte, die als männlicher Freier Penelopes verkleidet in Ithaka auf der Suche nach ihrem Mann erscheint. Auch in Noris' Penelope wird Ulisses Eifersucht durch die sich als Mann ausgebende Ariene entzündet, für die Penelope zu schwärmen vorgibt. Anders als in der Vorlage wird die Rolle der Nachkommenschaft von Ulisse und Penelope kurioserweise zur weiblichen Figur verändert. So heißt Telemaco nun Elvida, die aktiv an den Liebesintrigen partizipiert. Sie verliebt sich in den Feind ihrer Eltern namens Lutezio, und am Anfang der Oper wirft sie ihm einen Liebesbrief aus Penelopes Fenster. Darum denkt er, dass Penelope dessen Autorin sei. ${ }^{13}$

Noris entwickelt eine andere Art Komik als Minato. Im Gegensatz zu Lippio ist Gildo kein naiver Diener; er kann sich sogar weise und intelligent verhalten, wie z. B. in der Szene (I, 13), in der er den Zorn des Ulisse mit Vernunft zu besänftigen sucht. Noris verzichtet außerdem auf die bei Minato beliebten Wortwitze, und nutzt stattdessen das komische Potential dramatischer Ironie, wie etwa im Dialog zwischen Ariene/Arconte und Lutezio im zweiten Akt. Angesichts Lutezios hartnäckiger Verleugnung seiner früheren Liaison wirft Ariene ihm vor, Penelope als verheirateter Mann den Hof zu machen (Akt II, 9). Die Antwort Lutezios erzielt beim Publikum, dem die wahre Identität Arcontes bekannt ist, einen komischen Effekt:

Lu.: Prence, Arconte, sentite.

Tanto è ver c'habbia moglie

Quanto voi siete donna.

Ar.: Dunque se donna io fossi

Voi sareste marito?

Lu.: L'impossibil propongo.

Lu.: Prinz, Arconte, hören Sie.

Es ist genauso wahr, dass ich eine Gattin habe,

wie Sie ein Weib sind.

Ar.: Darum wären Sie Gatte,

wenn ich Weib wäre?

Lu.: Ich schlage das Unmögliche vor.

Trotz der unterschiedlichen Art der Witze ist die tragikomische Behandlung des Stoffes aus der Odyssee der gemeinsame Nenner von Penelope und Penelope la casta, wenngleich sie dramma per musica und nicht tragicommedia heißen: Die Mischung des Ernsten und des Komischen war in der italienischen Oper des Seicento die Norm, nicht die Ausnahme, und benötigte deswegen keine zusätzliche Erklärung in der Gattungsbezeichnung.

13 SCHULZE, Hendrick. Odysseus in Venedig, Frankfurt am Main: Peter Lang, 2004, S. 327ff. 
Einen tragikomischen Charakter besitzt - auch in diesem Fall ohne spezifische Kennzeichnung - ebenfalls Pietro Pariatis Theaterstück La casta Penelope. Es beruht offenbar auf Noris' Libretto und wurde 1707 im Teatro Ducale in Mailand erstmals aufgeführt. ${ }^{14}$ Vor seiner Berufung an den Wiener Kaiserhof im Jahre 1714 wirkte Pietro Pariati in Venedig als Mitarbeiter des jüngeren, doch erfahreneren Apostolo Zeno. Dabei transformierte er Opernlibretti in Schauspiele, die er selbst (Sesostri re d'Egitto), Apostolo Zeno (Griselda) oder Matteo Noris (Cajo Marzio Coriolano, Penelope la casta) geschrieben hatte. Letztere wurde 1707 für die Truppe von Luigi Riccoboni im Teatro Ducale in Mailand aufgeführt. Bereits der Titel bezieht sich auf Noris' Libretto als Vorlage, da das Adjektiv „casta“ (keusch) sonst in keinem anderen mir bekannten Libretto zu finden ist. Pariati nimmt im übrigen tiefgreifende Veränderungen der Handlung im Sinne einer Rationalisierung der Liebesbeziehungen vor: Die Figur des Lutezio mit den entsprechenden Intrigen wird ausgemerzt, Elvida wird gemäß der Odyssee wieder zu Telemaco, dessen Verlobte Doriclea die Rolle der sich als Mann ausgebenden Frau übernimmt. Pariatis Fassung gewinnt durch die zwei parallelen und erwiderten Liebesbeziehungen (Penelope - Ulisse, Doriclea - Telemaco) an Symmetrie.

Der übliche Diener Ulisses (namens Gradellino) spricht in Pariatis Tragikomödie aus dem Stegreif gemäß dem Usus der Commedia dell'arte: Im Text steht nur ein Canovaccio, das Gradellinos Reden und Agieren auf der Bühne zusammenfasst, während vollständige Dialoge für die anderen Rollen niedergeschrieben sind. Der Diener wird wieder zu einer eigentlichen komischen Figur, wie bei Minatos Penelope.

Neben dieser niedrigen Art der Komik übernimmt Pariati von Noris' Libretto gemäBigte dramatische Ironie, deren Quelle klar erkennbar in folgender Stelle liegt:

\section{Noris 1696 (Akt III, 8)}

Pen.: Sempre io lo sposo amai, benché lontano

Da me vagando ei visse.

Prencipe lo credete? ${ }^{15}$

Uli.: Io ‘l credo sì; ma no'l credeva Ulisse.

Pen.: Ich habe den Gatten immer geliebt, obwohl er

immer weit von mir entfernt lebte.

Prinz, glauben Sie es mir?

Uli.: Ich glaube es schon, aber Ulisse glaubte es nicht.

\section{Pariati 1707 (Akt III, 14)}

Pen:. [...] E tu ti rendi difficile a creder, che dal mio core mai un pensier contro lo sposo

14 Nach New Grove Dictionary of Music and Musicians und MGG2 wurde im selben Jahr im Mailänder Teatro Ducale eine Oper mit diesem Titel zu Musik von Andrea Stefano Fioré aufgeführt. Sowohl das Libretto als auch die Partitur gelten jedoch als verschollen.

15 In der 1685 Fassung sagt Penelope „Voi lo credete“ und singt daher einen fünfsilbigen statt eines siebensilbigen Verses; das Libretto für das Teatro di Tordinona (Musik von Giacomo Antonio Perti) von 1696 korrigiert diesen Fehler. 
uscisse?

Uli.: Il credo sì, ma no'l credeva Ulisse.

Pen.: Ist für dich schwer zu glauben, dass nie ein Gedanke gegen den Gatten meinem Herzen entwichen ist?

Uli.: Ich glaube es schon, aber Ulisse glaubte es nicht.

Ulisses Antwort „Il credo sì, ma no'l credeva Ulisse“ tritt mehrmals im Dialog als Refrain in Erscheinung ${ }^{16}$ und erzielt dadurch einen leicht komischen Effekt. Eine gewisse dramatische Ironie ergibt sich in diesem Fall aus dem Umstand, dass Penelope eigentlich nicht wissen sollte, dass sie mit Ulisse und nicht mit einem Freund von ihm spricht. Dieser Effekt wird zusätzlich durch das Wissen des Publikums gesteigert, dass Penelope Ulisse inzwischen erkannt hat und sein Spiel mit falscher Identität somit zwecklos geworden ist.

Pariati erweitert die Rolle der komischen Figuren in seiner Überarbeitung dieses Theaterstückes zur Tragicommedia per musica, die er fünfzehn Jahre später für den Wiener Kaiserhof schreibt (Musik von Francesco Bartolomeo Conti). Als Quelle tragikomischer Aktionen ersinnt Pariati dabei eine Rollenverwechslung zwischen dem Diener, hier Tersite genannt, und seinem Herrn Ulisse. Tersite verstellt sich als Prinz Antifate, ${ }^{17}$ und Ulisse als dessen Diener. Das Unbehagen Tersites in der Identität des Prinzen trifft auf Ulisses' Überschreitung der Grenzen seiner dienerischen Rolle, da er sich nicht zurückhalten kann und Penelope wegen ihrer vermuteten Untreue Vorwürfe macht. Die Rolle des Ulisses als Anti-Held wird durch die Rollenverwechslung noch stärker als in den vorigen Werken Minatos und Noris' hervorgehoben und charakterisiert diese Oper als tragicommedia per musica. ${ }^{18}$ Verstärkt wird die Komik außerdem durch die Hinzufügung eines weiblichen Gegenparts zum verkleideten Diener, mit entsprechenden Aufbau autonomer komischer Szenen am Ende jedes Aktes. Eine solche dégradation d'action wird zum parodistischen Echo auf die Aktionen des ernsten Liebespaares PenelopeUlisse. Es handelt sich dabei um eine bei Pariati beliebte dramaturgische Lösung, die er bereits 1707 in Anfitrione (seiner ersten Tragicommedia per musica) verwendet, und die

16 Solche Refrains im Dialog verwendete noch Metastasio, siehe u. a. Metastasios Ezio, Akt II, Szene 13. Piero Weiss unterstreicht die Virtuosität Metastasios in der Verwendung eines Verses als Kehrreim in einem Dialog („Caso speciale il virtuosismo con cui Metastasio sottolinea, rimando, un verso che ricorre a mo“ di intercalare nel corso d'una scena dialogata“, siehe WEISS, Piero. L'opera italiana nel '700, hrsg. von Raffaele Mellace, Roma: Astrolabio, 2013, S. 108.

17 Antifate (Antiphates) war König der riesenhaften Laistrygonen, welche die Flotte von Odysseus zerstörten. Dass Ulisse in der Oper diesen Namen für seinen Diener wählt, muss für das Publikum bereits komisch klingen.

18 Die Verkehrung der Rollen setzt Penelope in Bezug zu vorausgehenden tragikomischen Opern von Pariati und Conti, vor allem Il finto Policare (1716), bei dem der Prinz Policare seine Identität dem ungehobelten Turbone leiht und sich dafür als dessen Diener ausgiebt. Claudia Michels sieht die Rollenverwechslung in Zusammenhang mit der Karnevalisierung der Oper und dem Fasching als Anlass für die Aufführung dieser Tragicommedie, siehe MICHELS, Claudia. Karnevalsoper am Hofe Kaiser Karls VI. 1711-40, Wien: Universität Wien (Diss.), 2007. Eine Reduktion der Tragicommedia „ausschließlich als Hervorbingung des Karnevals“ würde aber andere Faktoren, u. a. die im vorliegenden Artikel vorgestellten Beziehungen mit der venezianischen Oper, zu Unrecht vernachlässigen (vgl. HIRSCHMANN, Konstantin. Die Tragicommedia Pietro Pariatis und Francesco Contis und ihre Rezeption in Norddeutschland am Beispiel des Don Chisciotte in Sierra Morena, Wien (Masterarbeit), 2016, S. 7. 
er von Molières Komödie Amphitryon übernommen hat: Das „ernste Dreieck“ Amphitryon-Alcmène-Jupiter wird von dem komischen Sosie-Cléanthis-Mercure konterkariert. Die Positionierung am Ende jedes Aktes der komischen Szenen ist dennoch eine alte Tradition, die bereits in venezianischen Opern der 1650er und 1660er von Aurelio Aureli und Nicolò Minato vorhanden ist. ${ }^{19}$

Der vorliegende Beitrag dient einer ersten Skizzierung der Kontinuitäts- und Neuerungselemente, welche Pariatis Tragicommedia per musica des frühen 18. Jahrhunderts definieren. Nachvollziehbar wird diese historische Einbettung durch die nachgezeichnete Verknüpfung zwischen den vier Penelope und den damit einhergehenden Entgrenzungen von Genres und Registern. Zukünftige Studien im Rahmen zweier SNF-Postdoc.Mobility Stipendien $^{20}$ werden die Tragicommedia per musica und ähnliche Genera mixta tiefergehend analysieren, um gemeinsame Merkmale in der dramaturgischen Gestaltung und der musikalischen Vertonung hervorzuheben. Auf diese Weise soll zur Schließung einer bisher vernachlässigten Forschungslücke beigetragen werden.

\section{Bibliography}

\section{Sources}

MINATO, Nicolò. Penelope, Wien: Cosmerovio, 1670.

NORIS, Matteo. Penelope la casta, Venezia: Nicolini, 1685.

PARIATI, Pietro. La casta Penelope, Milano: Ghisolfi, 1707.

PARIATI, Pietro. Penelope, Wien: Van Ghelen, 1724.

\section{Literature}

ANTONUCCI, Fausta - TEDESCO, Anna (eds). La comedia nueva e le scene italiane nel Seicento, Firenze: Olschki, 2016.

BIANCONI, Lorenzo. Music in the Seventeenth Century, Cambridge: Cambridge University Press, 1986.

COLAS, Damien und DI PROFIO, Alessandro (Hrsg.), D’une scène à l'autre, l'opéra italian en Europe, 2 Bde., Wavre, Mardaga, 2009.

DUBOWY, Norbert. Opere di Draghi in Italia? In „Quel novo Cario, quel divin Orfeo”: Antonio Draghi da Rimini a Vienna; atti del convegno internazionale, (Rimini, Palazzo Buonadrata, 5-7 ottobre 1998). Davide Daolmi - Emilio Sala (Hrsg.). Lucca: LIM, 2000.

19 FABBRI, Paolo. Il secolo cantante, Roma: Bulzoni editore, S. 219-20.

20 Das derzeitige Forschungsprojekt heißt Tragicommedia in musica. Entstehung, gattungsbezogene Merkmale und Wirkung der genera mixta vor Metastasio (ca. 1690-ca. 1730). Es setzt sich mit Libretti auseinander (siehe Webseite https://musikwissenschaft.univie.ac.at/projekte/aktuelle-projekte/tragicommedia-strafspiel-und-satire/), während das Folgeprojekt Tragicommedia per musica, Strafspiel und musikalische Vertonung des Satirischen in dramatischen Vokalwerken (ca. 1700-1750) den musikalischen Stil tragikomischer Werke hinterfragen wird. 
FABBRI, Paolo. Il secolo cantante, Roma: Bulzoni editore, 2003.

GENETTE, Gérard. Palimpseste: Die Literatur auf zweiter Stufe, Frankfurt am Main: Suhrkamp, ${ }^{6} 2012$. GIGLIUCCI, Roberto. Tragicomico e melodramma, Milano: Mimesis Edizioni, 2011.

HIRSCHMANN, Konstantin. Die Tragicommedia Pietro Pariatis und Francesco Contis und ihre Rezeption in Norddeutschland am Beispiel des Don Chisciotte in Sierra Morena, Wien (Masterarbeit), 2016.

KINTER, Barbara. Die Figur des Gracioso im spanischen Theater des 17. Jahrhunderts, München: Wilhelm Fink Verlag, 1978.

MICHELS, Claudia. Karnevalsoper am Hofe Kaiser Karls VI. 1711-40, Wien: Universität Wien (Diss.), 2007.

MURARO, Maria Teresa (ed.). L'opera italiana a Vienna prima di Metastasio, Firenze: Olschki, 1990 NOE, Alfred. Die italienische Literatur in Österreich, Wien: Böhlau Verlag, 2011.

NOE, Alfred. Nicolò Minato Werkverzeichnis, Wien: Der österreichischen Akademie der Wissenschaften, 2004.

SEIFERT, Herbert. Die Oper am Wiener Kaiserhof im 17. Jahrhundert, Tutzing: Schneider, 1985.

SCHULZE, Hendrick. Odysseus in Venedig, Frankfurt am Main: Peter Lang, 2004.

WEISS, Piero. L'opera italiana nel '700, ed. von Raffaele Mellace, Roma: Astrolabio, 2013. 
